\title{
Management of thyrotoxicosis occurring after surgery for Cushing's disease: a case series
}

\author{
Shenzhong Jiang ${ }^{1 \#}$, Chengxian Yang ${ }^{1 \#}$, Ming Feng ${ }^{1}$, Yong Yao ${ }^{1}$, Kan Deng ${ }^{1}$, Bing Xing ${ }^{1}$, Lin Lu ${ }^{2}$, \\ Huijuan Zhu' ${ }^{2}$, Renzhi Wang ${ }^{1}$, Xinjie Bao ${ }^{1}$ \\ ${ }^{1}$ Department of Neurosurgery, Pituitary Center, Peking Union Medical College Hospital, Chinese Academy of Medical Sciences and Peking Union \\ Medical College, Beijing, China; ${ }^{2}$ Key Laboratory of Endocrinology of National Health Commission, Department of Endocrinology, Peking Union \\ Medical College Hospital, Chinese Academy of Medical Sciences and Peking Union Medical College, Beijing, China \\ Contributions: (I) Conception and design: All authors; (II) Administrative support: R Wang, X Bao; (III) Provision of study materials or patients: \\ M Feng, Y Yao, K Deng, B Xing, L Lu, H Zhu, R Wang, X Bao; (IV) Collection and assembly of data: S Jiang, C Yang; (V) Data analysis and \\ interpretation: All authors; (VI) Manuscript writing: All authors; (VII) Final approval of manuscript: All authors. \\ "These authors contributed equally to this work. \\ Correspondence to: Xinjie Bao; Renzhi Wang. Department of Neurosurgery, Pituitary Center, Peking Union Medical College Hospital, \\ Chinese Academy of Medical Sciences and Peking Union Medical College, No. 1 Shuaifuyuan, Dongcheng District, Beijing 100730, China. \\ Email: baoxinjie1@pumch.cn; wangrz@126.com.
}

Background Thyrotoxicosis is a rare complication of surgery for Cushing's disease (CD). In clinical practice, given the rarity of this condition, patients may be misdiagnosed, and thus not treated appropriately. This study describes the clinical features and management of thyrotoxicosis in this context.

Methods: We retrospectively screened a pituitary database for CD patients who underwent surgery at Peking Union Medical College Hospital between 2010 and 2017. The electronic medical records and documentation of 732 patients were searched in order to identify instances of thyrotoxicosis.

Results: Fourteen patients developed postoperative thyrotoxicosis after CD remission, representing $1.9 \%$ of all the CD patients who underwent surgery and $2.5 \%$ of the CD patients who were in remission after surgery. The onset of thyrotoxicosis occurred at a median of 3.5 months after surgery. At the onset, 13 patients $(92.9 \%)$ were on a maintenance dose of hydrocortisone and were managed using a higher dose of hydrocortisone. One patient had normal adrenal function and was managed by observation first, then using anti-thyroid drugs. Thyrotoxicosis was transient in all but one patient, and lasted $1.9 \pm 0.7$ months (range, $1-$ 3.2 months).

Conclusions: Thyrotoxicosis is a rare complication that develops during the recovery period following surgery for CD. It occurs only after the surgical resolution of hypercortisolism, and in particular alongside adrenal insufficiency during the 4 months following CD remission. Awareness of this situation should be promoted among physicians, and patients should be managed on the basis of the clinical setting and manifestation.

Keywords: Thyrotoxicosis; Cushing's disease (CD); hydrocortisone replacement; adrenal insufficiency

Submitted Jan 22, 2021. Accepted for publication Mar 18, 2021.

doi: 10.21037 /gs-21-49

View this article at: http://dx.doi.org/10.21037/gs-21-49 


\section{Introduction}

Cushing's disease (CD), the most common form of endogenous Cushing's syndrome (comprising approximately $70 \%$ of cases), is caused by pituitary corticotropin (ACTH)secreting tumors, which induce chronic hypercortisolemia leading to a reversible state of immunosuppression (1). Hypercortisolism is associated with higher risks of metabolic, musculoskeletal, immunological, and neuropsychiatric complications, which result in significant morbidity and mortality. Therefore, CD requires early diagnosis and treatment, and transsphenoidal surgery (TSS) is currently the treatment of choice $(2,3)$. The reported remission rates are between $68 \%$ and $98 \%$ (4).

Some have studied the relationship between thyroid abnormalities and hypercortisolism in either active phase or upon resolution. Hypercortisolism is found to be associated with an increased prevalence of nodular goiter, while serum anti-thyroid antibodies are similar between CD patients and the general population (5). Regarding thyroid function test (TFT), generally, inhibition of thyroid stimulating hormone (TSH) secretion was demonstrated in patients with longterm exposure to high cortisol levels, while serum free triiodothyronine (T3) and free thyroxine (T4) levels were similar compared to controls $(5,6)$. Hypothyroidism with normal/low TSH and low T3 and T4 levels is also noticed in CD patients (7). Of note, normal TSH and depressed serum T3 levels suggestive of sick euthyroid condition could also be seen in patients with hypercortisolism, which might be mistaken for hypothyroidism but do not necessitate therapy (8). Incidentally, co-existing cases of hypercortisolism and thyrotoxicosis are extremely rare (9-13), with only one case of CD reported coexisting with Graves' disease (GD) (13).

Studies about conditions arising from rebound in immunity upon CD remission mainly focus on thyroid autoimmune diseases. Despite this, the development of thyrotoxicosis related to thyroid autoimmunity after surgery for CD has been reported in only a few patients. Takasu et al. (14) reported that one patient developed transient thyrotoxicosis, which was attributed to autoimmune thyroiditis, following successful treatment of their CD. Colao et al. (5) briefly mentioned two cases of thyrotoxicosis resulting from thyroiditis after surgery. In addition, a further six patients were reported to have developed thyrotoxicosis because of Graves' disease after surgery for CD (13,15-18). Apart from this, several isolated cases of other conditions upon resolution of hypercortisolism have also been reported and call attention to a wider spectrum of immune dysfunctions, including rheumatoid arthritis (19), seronegative arthritis (20), systemic lupus erythematous (21), etc.

The mechanism underlying the development of thyrotoxicosis in patients with CD that is surgically resolved has not been determined, but a rebound in immunity after disease remission is considered to play a crucial role in the process (1).

To date, there have been few publications regarding thyrotoxicosis occurring after $\mathrm{CD}$ remission. In the present study, we report a series of cases of thyrotoxicosis that developed after surgery for $\mathrm{CD}$, discuss their clinical features, and describe our experience with the management of such cases.

We present the following article in accordance with the STROBE reporting checklist (available at http://dx.doi. org/10.21037/gs-21-49).

\section{Methods}

\section{Patients}

Using the pituitary database of Peking Union Medical College Hospital (PUMCH), we retrospectively screened 732 patients who were diagnosed with $\mathrm{CD}$ and underwent TSS at PUMCH between January 2010 and December 2017. In this cohort, immediate remission of CD after surgery was achieved in 564 patients, whereas 168 patients had persistent CD (defined by the failure of remission after surgery and the requirement of further additional treatments, including pituitary radiotherapy, adrenal surgery, and/or medical therapy). For all the patients, records relating to all their clinic visits were searched to identify instances of thyrotoxicosis (characterized by the clinical manifestations of inappropriately high thyroid hormone action in tissues generally due to inappropriately high tissue thyroid hormone levels). Patients with known thyroid disease that require active therapy at the time of $\mathrm{CD}$ diagnosis were excluded. A total of 14 patients were found to have developed thyrotoxicosis after TSS for ACTHdependent pituitary adenoma and were selected for further analysis. All of the 14 patients were in remission of their CD before the onset of thyrotoxicosis, and none had persistent CD. Thus, $1.9 \%(14 / 732)$ of all the CD patients who underwent surgery, and $2.5 \%$ (14/564) of CD patients who were in remission after surgery developed thyrotoxicosis. We evaluated their clinical course from before surgery to at 
least 24 months afterwards.

Besides, a control group, comprising 732 subjects operated in our pituitary center for clinically nonfunctioning pituitary adenomas (NFPA) during the same period, matched by age and gender with $\mathrm{CD}$ patients, was used for this study. Controls were unselected. All denied having known thyroid diseases that required active therapy. In the control group, TFTs panel were obtained both before and after TSS. The study was conducted in accordance with the Declaration of Helsinki (as revised in 2013). This study was approved by the Ethics Committee of PUMCH (JS2288) and individual consent for this retrospective analysis was waived.

\section{Diagnosis of CD}

The diagnosis of CD was based on the clinical symptoms and signs, imaging, and hormone assays (22).

(I) A pituitary adenoma was found by magnetic resonance imaging (MRI). Microadenomas were defined as the largest diameter $<10 \mathrm{~mm}$, while macroadenomas were defined as the largest diameter $\geq 10 \mathrm{~mm}$.

(II) There was a high 24-hour urine free cortisol (24h-UFC), and high serum plasma cortisol (normal range: $4.0-22.3 \mu \mathrm{g} / \mathrm{dL}$ ) and ACTH concentrations.

(III) A combined low-dose and high-dose dexamethasone suppression test showed a failure of 24h-UFC suppression after the low dose but a $>50 \%$ reduction in the baseline level after the high dose.

(IV) Bilateral inferior petrosal sinus sampling was performed to support the diagnosis of CD if necessary.

All selected 14 patients fulfilled the above-mentioned criteria for CD diagnosis.

\section{Remission of CD}

All the patients underwent TSS for the treatment of CD. Immediate remission was defined as a morning serum cortisol concentration $<5 \mu \mathrm{g} / \mathrm{dL}(138 \mathrm{nmol} / \mathrm{L})$ within 7 days after surgery (23).

\section{Postoperative management}

Hormone replacement therapy was initiated once immediate remission had been achieved, with intravenous hydrocortisone $100 \mathrm{mg}$ twice daily for 3 days and then oral hydrocortisone $60 \mathrm{mg}$ daily. After discharge, the dose of hydrocortisone was gradually tapered by $10 \mathrm{mg}$ per week until it reached $10 \mathrm{mg}$ per day, and was discontinued after evaluation of pituitary-adrenal function. Typically, a one-totwo-month course of hydrocortisone was required. Pituitary function was assessed 1, 3, and 12 months after surgery, and annually thereafter, to confirm long-term remission of $\mathrm{CD}$, when thyroid function was also assessed.

Serum free thyroxine (FT4), free triiodothyronine (FT3) and thyroid stimulating hormone (TSH) levels were measured by immunoassays (Advia Centaur XP Automatic Chemiluminescence Immunoassay Analyzer; Siemens Healthineers, Erlangen, Germany). Thyroid ultrasonography, thyroglobulin antibody (TgAb), thyroid peroxidase antibody (TPOAb), and thyrotropin receptor antibody (TRAb) assays were performed as appropriate. The TgAb, TPOAb and TRAb measurements were performed using Roche kits and included reagents (Cobas e601, Roche Diagnostics, Mannheim, Germany). Normal reference ranges are as follows: FT4: $0.81-1.89 \mathrm{ng} / \mathrm{dL}$; FT3: 1.8 $4.1 \mathrm{pg} / \mathrm{mL}$; TSH: 0.38-4.34 $\mu \mathrm{IU} / \mathrm{mL}$; TgAb: <115 IU/mL; TPOAb: <34 IU/mL; TRAb: <2.5 IU/L.

\section{Statistical analysis}

In this descriptive study, categorical variables are expressed as percentages. Normally distributed continuous variables are expressed as mean \pm standard deviation (SD), and nonnormally distributed variables as median and interquartile range (IQR). The comparison between the numerical data were performed by Student's $t$-test for unpaired or paired data. The

$\mathrm{P}$ values were given for the analysis. Significance was set at $5 \%$.

\section{Results}

\section{Patient characteristics}

The characteristics of the selected patients are shown in Table 1. The median age of these 14 patients when CD was diagnosed was 32.5 years (IQR $=27.5-37.5$ years, range, 16-80 years), and they had had the disease for a median of 3 years (IQR $=1.9-7.3$ years, range, $1-8$ years) prior to surgery. All of the patients were women. 12 patients $(85.7 \%)$ demonstrated a microadenoma, and $2(14.3 \%)$ demonstrated a macroadenoma. One patient (number 4) 
Table 1 Main features in the 14 studied patients before surgery

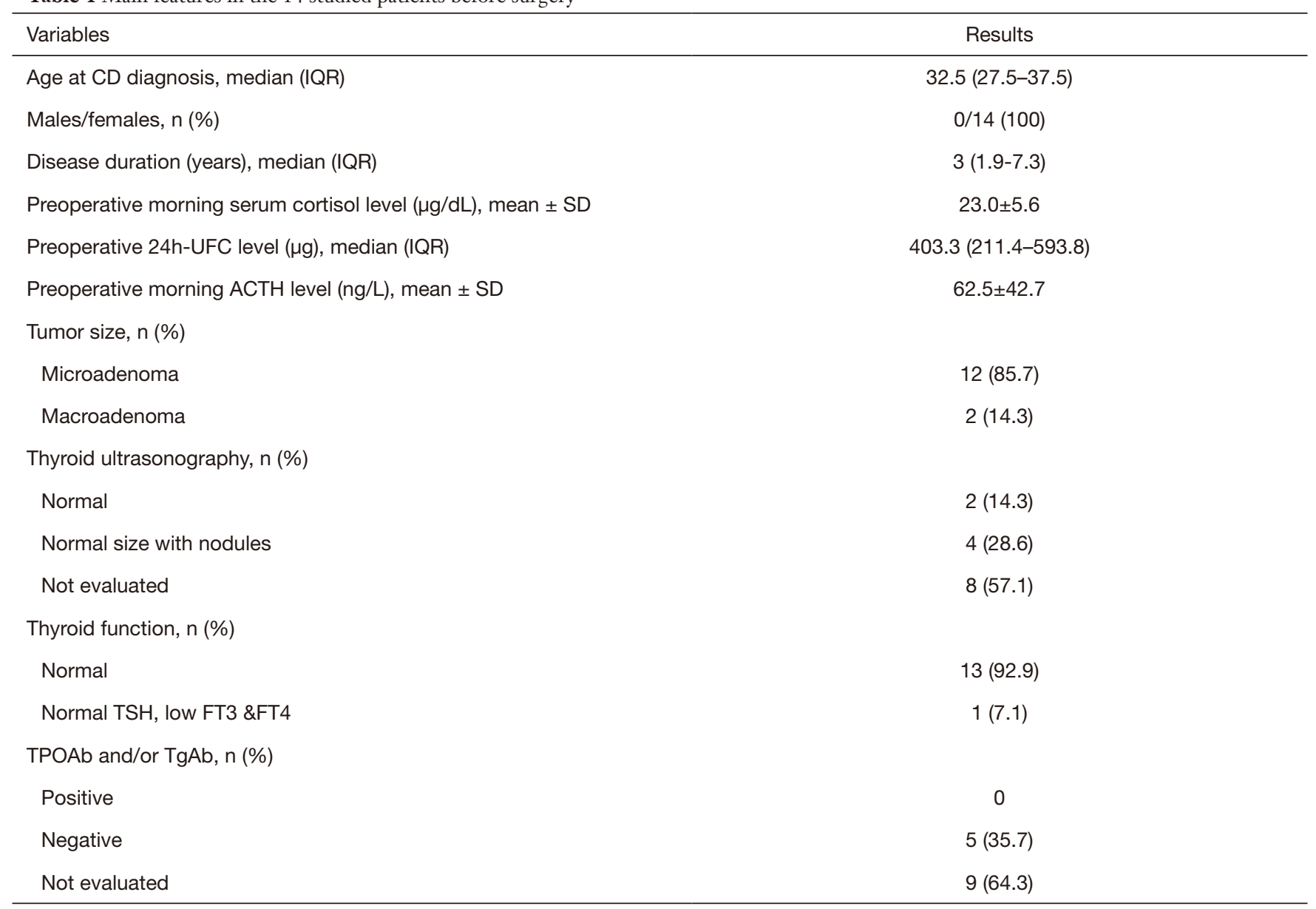

CD, Cushing's disease; ACTH, adrenocorticotropin; UFC, urinary free cortisol; IQR, interquartile range; SD, standard deviation; FT4, free thyroxine; FT3, free triiodothyronine; TSH, thyroid stimulating hormone; TPOAb, anti-thyroid peroxidase antibody; TgAb, anti-thyroglobulin antibody; TRAb, anti-thyrotropin receptor antibody. Hormonal normal values, cortisol: 4.0-22.3 $\mu \mathrm{g} / \mathrm{dL} ; \mathrm{ACTH}$ : 0-46.0 pg/mL; 24h-UFC: $12.3-103.5 \mu \mathrm{g} / 24 \mathrm{~h}$.

had central hypothyroidism and was taking levothyroxine (L-T4) replacement at the time of surgery. Of the five patients tested for thyroid antibodies within one week before surgery, none were positive (see Table S1 for preoperative details).

\section{Comparison between $C D$ and control groups}

In the NFPA control group, we detected ten patients (1.4\%) who took L-T4 after TSS due to postoperative hypothyroidism, while no patients were found to have TFTs of low TSH, high FT4 and/or high FT3, nor had any of them reported thyrotoxic symptoms. While in CD group, a total of 25 patients $(3.4 \%)$ were detected to have abnormal thyroid function after surgery compared with $1.4 \%$ of controls $(\mathrm{P}=0.0154)$, and $1.9 \%$ of all the $\mathrm{CD}$ patients developed postoperative thyrotoxicosis compared with none in the control group $(\mathrm{P}<0.0001)$.

\section{Clinical course}

After surgery, three patients had central hypothyroidism before thyrotoxicosis developed. At the onset of thyrotoxicosis, one patient had already discontinued hydrocortisone, but 13 patients $(92.9 \%)$ had not been able to taper off their dose because of adrenal insufficiency, and were on a maintenance dose of $10 \mathrm{mg}$ per day. All patients had suppressed TSH, and elevated FT4 and/or FT3, and had more than one abnormal thyroid function measurement. 14 patients developed symptomatic thyrotoxicosis. 
Table 2 Clinical profiles of 14 patients with postoperative thyrotoxicosis after surgery for Cushing's disease

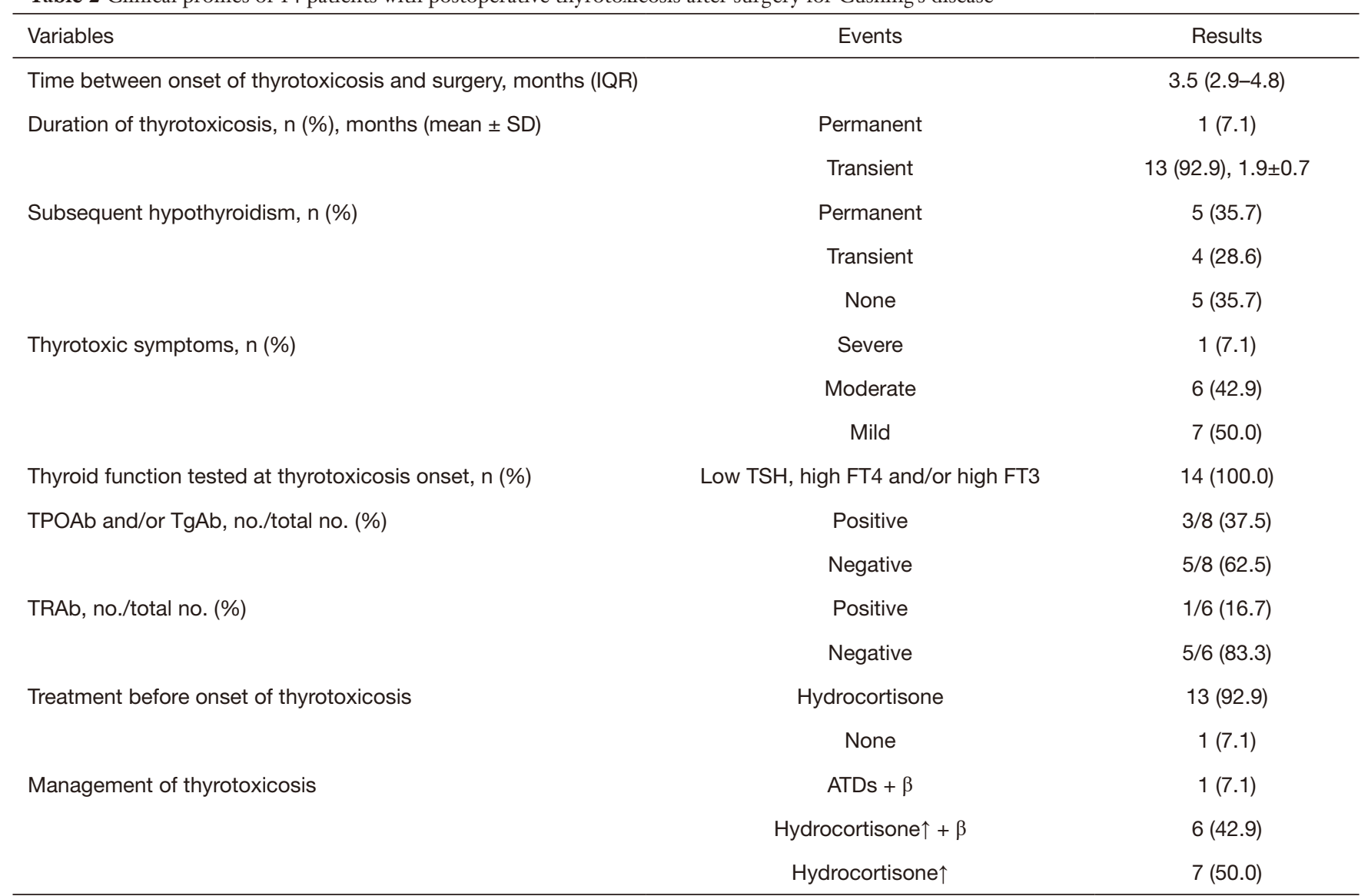

IQR, interquartile range; SD, standard deviation; FT4, free thyroxine; FT3, free triiodothyronine; TSH, thyroid stimulating hormone; TPOAb, anti-thyroid peroxidase antibody; TgAb, anti-thyroglobulin antibody; TRAb, anti-thyrotropin receptor antibody; ATDs, anti-thyroid drugs; $\beta$, $\beta$-blockers; Hydrocortisone $\uparrow$ : higher dose of hydrocortisone.

Clinically, one patient had severe symptoms with significant weight loss, palpitation, etc. and needed persistent therapy, 6 patients had moderate symptoms that required a limited course of $\beta$-blockers, the other 7 patients developed mild symptoms that did not require $\beta$-blocker therapy. The severity of thyrotoxicosis symptoms did not correlate with post-op cortisol levels $(\mathrm{P}=0.6307)$. Anti-thyroid antibody titers were measured in eight of the 14 patients, and TPOAb and/or TgAb tests were positive in three patients and negative in five patients. Thyrotoxicosis was diagnosed a median of 3.5 months (IQR $=2.9-4.8$ months, range, 1.2 7.3 months) after surgery for CD. One patient was observed first, then prescribed anti-thyroid drugs (ATDs), 13 patients with mild to moderate thyrotoxic symptoms were prescribed a higher dose of hydrocortisone. The thyrotoxicosis was transient in all but one patient (number 1), lasting from 1 to 3.2 months (mean \pm SD, $1.9 \pm 0.7$ months). Of the 13 patients with transient thyrotoxicosis, subsequent persistent hypothyroidism developed in two patients (numbers 5, 14), pre-existing hypothyroidism persisted in three patients (numbers 2-4), and four patients (numbers 6-9) had transient hypothyroidism (Table 2 and Figure 1). (also see Table S2 for post-operative details).

\section{Case series}

The clinical course of 14 patients is summarized below, and thyroid function test results of 5 representative patients (numbers 1, 2, 5, 9, 14) is summarized in Figure 2.

Patient 1 was a 33-year-old woman who was diagnosed with $C D$ and underwent curative surgery. After surgery, she was placed on glucocorticoid replacement therapy, with instructions to taper the dose and discontinue her medication 1.5 months after surgery. She did not experience 


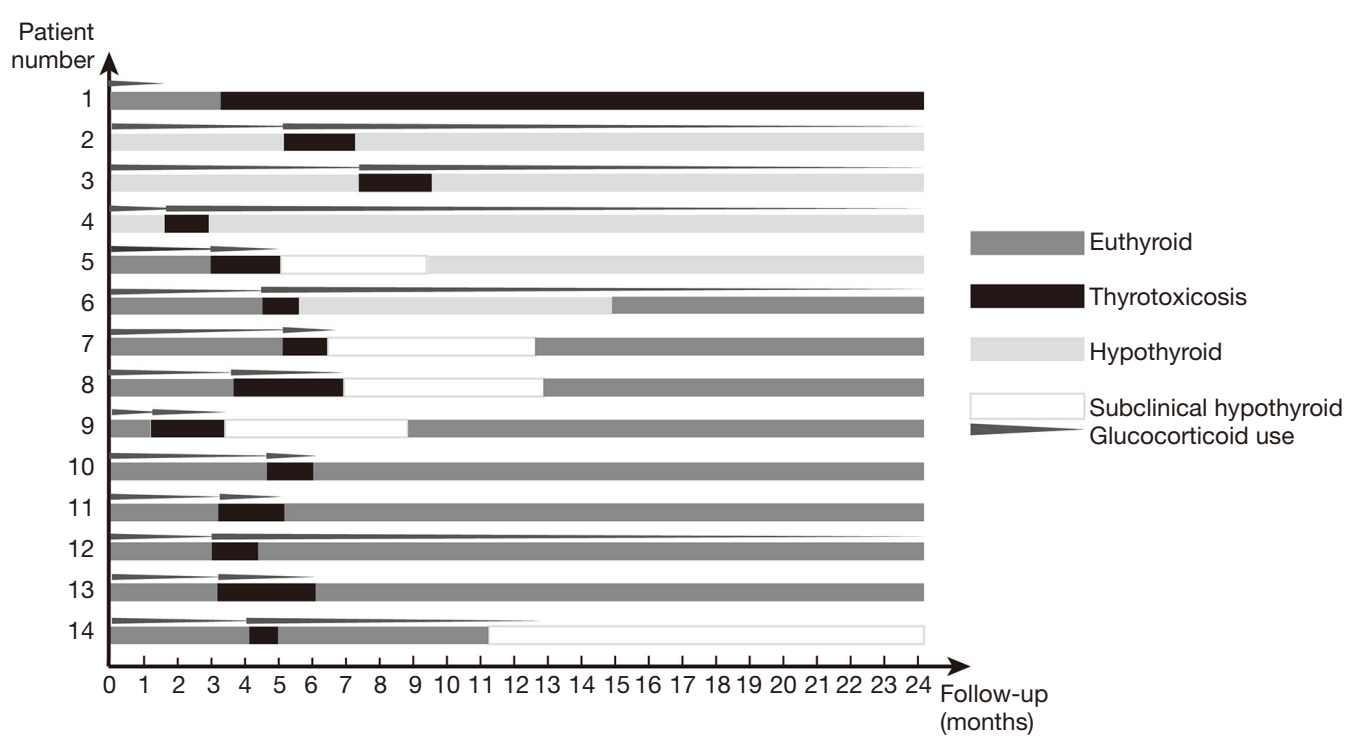

Figure 1 Clinical courses of thyroid function and glucocorticoid use in 14 patients after surgery for Cushing's disease. The horizontal axis shows the follow-up time after surgery for Cushing's disease in months.

adrenal insufficiency after that. Three months after surgery, during a routine follow-up examination, her laboratory work-up showed slightly high serum thyroid hormone concentrations (FT4: $1.647 \mathrm{ng} / \mathrm{dL}$; FT3: $5.59 \mathrm{pg} / \mathrm{mL}$; TSH: $0.047 \mu \mathrm{IU} / \mathrm{mL}$ ). At the same time, her morning plasma cortisol (08:00) concentration was $14.47 \mu \mathrm{g} / \mathrm{dL}$. She reported occasional fine finger tremor. No special treatment was prescribed as the clinical features of her condition were mild. Three months later, however, she gradually developed worsening thyrotoxic symptoms, including excessive perspiration, palpitation, finger tremor, an enlarged thyroid, and exophthalmos. Thyroid ultrasonography showed an increase in the size of the entire gland. Her FT4 and FT3 concentrations were abnormally high (FT4: $8.171 \mathrm{ng} / \mathrm{dL}$; FT3 $>20 \mathrm{pg} / \mathrm{mL}$ ) and her TSH was undetectable $(<0.008 \mu \mathrm{IU} / \mathrm{mL})$. She was positive for TRAb (30.82 IU/L, normal range, $<2.5 \mathrm{IU} / \mathrm{L})$. Therefore, GD was diagnosed and she was prescribed metoprolol and methimazole to manage her hyperthyroidism. However, this was unable to fully control her hyperthyroidism. At a follow-up examination 3 years after the initiation of treatment, she remained hyperthyroid, with a high TRAb titer of 10.93 IU/L, and had mild inactive Graves' ophthalmopathy. She was continuing to take methimazole $10 \mathrm{mg}$ three times daily.

Three patients (numbers 2-4) were taking L-T4 replacement therapy $(50 \mu \mathrm{g}$ each) after surgery because of postoperative central hypothyroidism. None of these patients were able to discontinue their glucocorticoid replacement because of adrenal insufficiency. All three patients developed signs and symptoms of thyrotoxicosis after surgery (after 5.1, 7.3, and 1.6 months, respectively). Evaluation of their thyroid function showed low TSH and high FT4 concentrations. At the same time, their morning plasma cortisol concentrations before they administered their hormone replacement doses were low $(0.2,0.5$, and $5.04 \mu \mathrm{g} / \mathrm{dL}$ ). L-T4 was temporarily discontinued and a double dose of hydrocortisone was administered, which resulted in gradual clinical and biochemical improvement, over 2.1, 2.1, and 1.2 months, respectively. Their FT4 concentrations normalized, followed by their symptoms of hypothyroidism. Subsequently, after experiencing transient thyrotoxicosis, all were placed on L-T4 replacement therapy $(75,50$, and $100 \mu \mathrm{g}$ each, respectively) to maintain their FT4 concentrations slightly above the middle of the normal range. All were also maintained on hydrocortisone at the lowest possible dose to prevent adrenal insufficiency.

Nine patients (numbers 5-13) developed transient thyrotoxicosis 1.2-5.1 months after the resolution of their $\mathrm{CD}$, and none were prescribed L-T4 after surgery. All were unable to taper off their hydrocortisone dose at the onset of thyrotoxicosis. All presented with mild-tomoderate symptoms of thyrotoxicosis. These patients were prescribed a double dose of hydrocortisone, and for those 

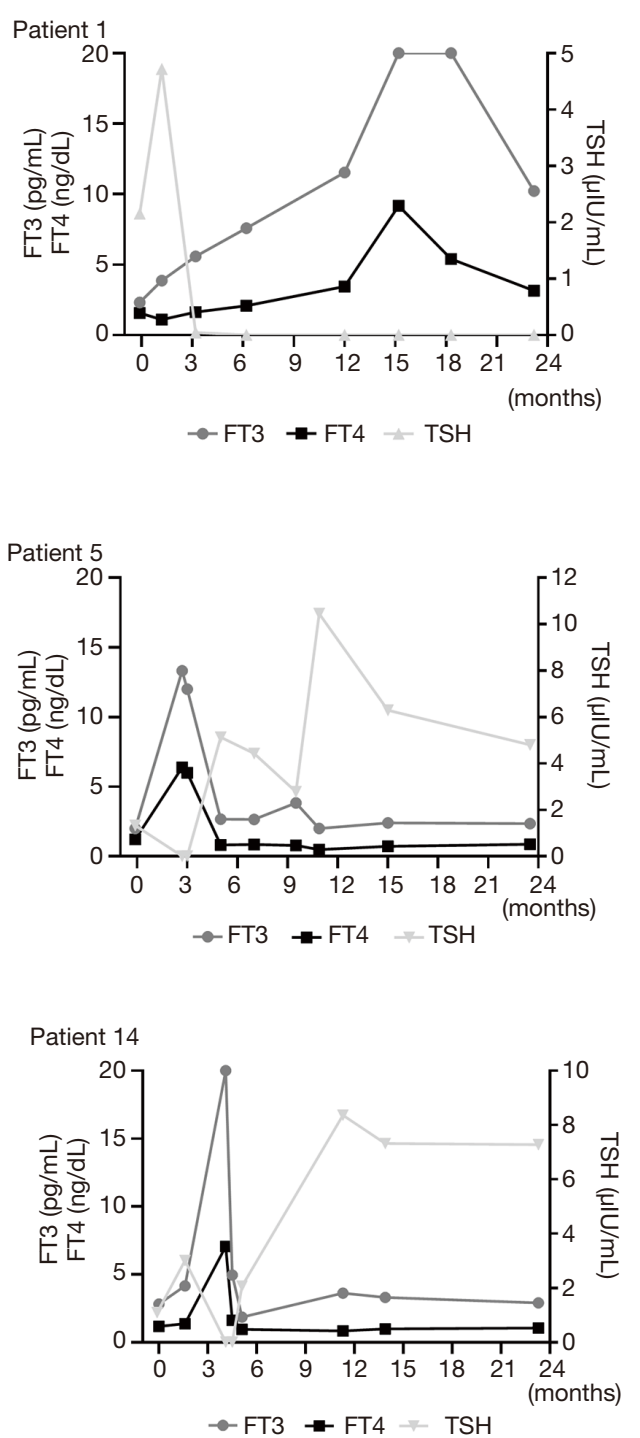
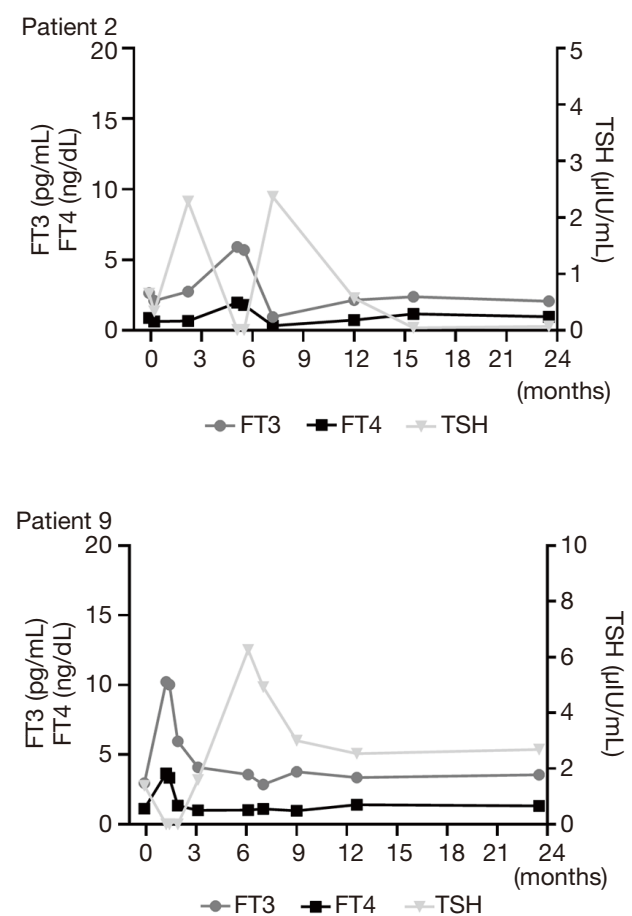

Figure 2 Thyroid function test results over time of Patients 1, 2, 5, 9, 14. The left vertical axes show values of free T3 (FT3) and free T4 (FT4), the right vertical shows (thyroid-stimulating hormone) TSH levels, and the horizontal axis shows the time after surgery in months.

with moderate symptoms, we also prescribed metoprolol as required. After this, their symptoms gradually resolved. Of the 9 patients, transient subclinical or overt hypothyroidism occurred in four patients (numbers 6-9), and one (number 5) developed permanent hypothyroidism and was prescribed L-T4 67.5 $\mu \mathrm{g}$ per day.

Patient 14 was a 35 -year-old woman who developed moderate neck pain and malaise, accompanied by a slight fever, during the fourth month following successful surgery. Her thyroid was tender on palpation. Further work-up revealed thyrotoxicosis, a high erythrocyte sedimentation rate of $60 \mathrm{~mL} / \mathrm{h}$, and a high thyroglobulin concentration, but negative thyroid autoantibodies. Ultrasonography showed that the thyroid was diffusely hypoechogenic, with low blood flow. On this basis, a diagnosis of de Quervain's Thyroiditis (DQT) was made. She was prescribed $15 \mathrm{mg} /$ day prednisolone, and experienced immediate relief of her neck pain, then the dose was tapered down, with continuing clinical improvement. She returned to her former hydrocortisone dose of $10 \mathrm{mg}$ per day 1 month later. Her FT3 and FT4 concentrations normalized and then her TSH concentration increased. Seven months after the onset of thyrotoxicosis, the patient developed subclinical hypothyroidism and was managed by the administration of 
L-T4 $50 \mu g$ per day.

\section{Discussion}

In the present report we have described 14 patients who developed thyrotoxicosis after surgery for CD and their management, and shown that thyrotoxicosis is rare in this context (prevalence of $1.9 \%$ ). At the onset of thyrotoxicosis, 92.9\% of the patients were on a maintenance dose of hydrocortisone, because of adrenal insufficiency, and were managed using a higher dose of hydrocortisone. One patient had resumed normal pituitary-adrenal function, and was managed by observation first, then using anti-thyroid drugs.

The development of thyrotoxicosis after surgery can be attributed to Cushing's disease per se, and is due to the remission of hypercortisolism, because none of the patients who were not in remission of their CD developed thyrotoxicosis. Although it is known that latent, subclinical hyperthyroidism and even autoimmunity with euthyroidism may be switched to overt hyperthyroidism by stress (surgery included) (24), surgery did not play a pivotal role in the process as none in the control group who underwent the same surgery developed thyrotoxicosis.

Hypercortisolism is known to exert an inhibitory effect on the immune system (25). Immune rebound occurs after the remission of hypercortisolism, and this is considered to be responsible for the development or exacerbation of autoimmune diseases, with thyroid autoimmunity being the most commonly reported form (1). The cortisol levels remained stable both before and after surgery in controls and in those who were not cured of CD. Therefore, their immune system was not affected or changed perioperatively. Only patients with cured CD experienced an abrupt decline of cortisol levels, which led to the rebound autoimmunity during the remission phase of $\mathrm{CD}$, possibly triggering the onset of thyroid autoimmune diseases.

It is interesting to note that all patients that developed thyrotoxicosis after cure of CD are females. As in adult CD patients, females are more frequently affected than males with a female/male ration of 3-8:1 (26). In our cohort, the proportion of female patients in CD is $80.7 \%$. In addition, female preponderance exists in autoimmune thyroid disease (27). Therefore, it is not strange that all the 14 patients that developed postoperative thyrotoxicosis are females.

Thyroid autoimmune diseases (GD and painless thyroiditis) are the major causes of thyrotoxicosis, according to the few published cases $(5,13-18)$, although one case of de Quervain's thyroiditis has been reported (28). However, the underlying mechanism for the development of thyroid autoimmunity in patients showing remission of $\mathrm{CD}$ has not been established.

The development of GD after the successful treatment of CD was first described by Morita et al. (16). In patient 1 , normal pituitary adrenal function had been restored when thyrotoxicosis developed 3 months after surgery. The patient was not treated at first, because the clinical features of her condition were mild, but she was told to report any changes in her symptoms. It was only when the signs of thyrotoxicosis worsened that GD was suspected and was later confirmed by the identification of TRAb. She has been administering ATDs for a long period of time since then and has achieved clinical improvement, but has not achieved complete remission. We speculate that GD might have been masked by the development of CD in this patient, because glucocorticoids can alter immune responses and suppress the production of autoantibodies (29). It is likely that the underlying GD was revealed because of an immune rebound following the abrupt reduction in cortisol concentration resulting from the surgery.

Painless thyroiditis can also develop after the remission of $\mathrm{CD}$. Painless thyroiditis is characterized by transient thyrotoxicosis, which is sometimes followed by hypothyroidism, then recovery, but $20 \%$ of patients develop permanent hypothyroidism (30). The clinical courses in the present cases (patient numbers 2-13) were identical to those expected for painless thyroiditis. Because of the transient nature of the thyrotoxicosis and its short duration, though thyroid autoantibody titers were not measured in all the patients, we speculate that the thyrotoxicosis was the result of painless thyroiditis as we have ruled out other possible causes, including syndrome of inappropriate secretion of TSH, impact of steroids, toxic multinodular goiter, use of medications or over the counter drugs.

Colao et al. (5) showed that there was a higher prevalence of autoimmune thyroiditis in CD patients (7/20) after the resolution of hypercortisolism than before, and two of their patients developed transient thyrotoxicosis because of painless thyroiditis. However, in this study, pituitaryadrenal function recovered in most of the studied patients, whereas in the present study it had not fully recovered in up to $95 \%$ of the patients when thyrotoxicosis developed. Tapering of the hydrocortisone dose in these patients is difficult, because every attempt to taper it results in the induction of symptoms, such as anorexia, nausea, and 
vomiting; therefore, these patients took maintenance doses of $10 \mathrm{mg}$ hydrocortisone once each morning, and did not experience symptoms related to adrenal insufficiency. With regard to management, we prescribed a double dose of hydrocortisone for those patients who were still taking a maintenance dose, and then adopted a "wait and see" approach. In some patients with moderate thyrotoxic symptoms, supportive therapy, such as $\beta$-blockers, was used.

Notably, thyrotoxicosis developed in the context of central hypothyroidism in three patients (numbers 2-4). All three of the present patients could not taper off their hydrocortisone dose because of the presence of hypoadrenocorticism. All had been on L-T4 replacement therapy before the onset of thyrotoxicosis. This begs the question of whether the thyrotoxicosis might have been induced by L-T4 administration. The discontinuation or a reduction in the dose of L-T4 is usually the only treatment required for iatrogenic hyperthyroidism, and serum T4 concentrations fall in about $50 \%$ of patients within a week of this (31). However, it took 1-2 months for the T4 concentrations to fall into the normal range in the present cases. Moreover, all the patients required a higher dose of L-T4 afterwards than before the onset of thyrotoxicosis to maintain a free $\mathrm{T} 4$ concentration in the middle of the normal range. This is consistent with our presumption of painless thyroiditis, because a smaller dose would be required if the thyrotoxicosis had been caused by L-T4 administration.

It has been recommended that thyroid function should be assessed during the 6 months following the remission of CD (5). However, the present case series demonstrates that thyrotoxicosis develops a median of 3.5 months after CD remission. Therefore, we recommend assessing this during the first 4 months following remission. Of note, manifestation of thyrotoxicosis occurred more than 6 months after adenoma removal in patient 3. Yamakita et al. (32) described a patient in whom thyrotoxicosis occurred 9 months after the adrenalectomy when the replacement dose of prednisolone was tapered to $5 \mathrm{mg}$ per day. Such a delayed appearance of thyrotoxicosis may be due to a gradual tapering of glucocorticoid dose. Together with our case, we suggest that thyrotoxicosis occurred not only with a rapid but also with a slow decline of glucocorticoid hormones.

In the literature, GD accounted for over half of the postoperative cases of thyrotoxicosis $(13,15-18)$, but in contrast, only one case of GD was diagnosed in the present study. The appropriate treatment of thyrotoxicosis is dependent upon the underlying etiology. For instance, if painless thyroiditis were to be treated like GD, there would be adverse effects. Thus, in order to avoid inappropriate treatment, our findings suggest that an algorithm should be used for the management of thyrotoxicosis in patients in remission from CD. First and foremost, it should be determined whether adrenal function has recovered after surgery, and if not, whether sufficient glucocorticoid replacement therapy is being administered. Second, the dose of hydrocortisone should be increased in patients with adrenal insufficiency to meet their physiological needs. If adrenal function has recovered or sufficient replacement of hydrocortisone is being administered, a "wait and see" strategy should be adopted, regardless of the cause of the thyrotoxicosis. Third, the symptoms and signs of thyrotoxicosis in most patients subside with time, but if they worsen, some of these, such as neck pain or exophthalmos, should direct us toward possible diagnoses of DQT or GD. Then, for example, the measurement of a TRAb titer would confirm a diagnosis of GD. Such patients can be managed as per standard guidelines. Supportive care, such as the administration of $\beta$-blockers, should be used in patients with significant thyrotoxic symptoms. Follow-up is also required to identify hypothyroidism.

The strength of the present study was the large number of CD patients included, which was possible because PUMCH is one of the largest centers for CD treatment in China. The main limitation of the study was its retrospective nature. In some cases, clinical data are missing including thyroid antibody and ultrasound, and patients were not consistently tested for thyroid autoimmunity. Therefore, to further elucidate the pathophysiological mechanism of the phenomenon, a prospective study regarding complete thyroid antibodies, thyroid ultrasound, scintigraphy, cytological examinations would be helpful in CD patients. In addition, the control group in our study is matched by age and gender, rather than by hormones or tumor size before surgery, this would cause bias in the study.

In conclusion, we have shown that thyrotoxicosis is a rare complication during the recovery period following TSS for CD and that it occurs only after the resolution of hypercortisolism, and particularly in the context of adrenal insufficiency during the 4 months following CD remission. It is important to promote awareness of this potential complication among physicians. The most appropriate means of managing patients should be determined on the basis of the clinical setting and the clinical manifestation of the thyrotoxicosis. 


\section{Acknowledgments}

We are grateful to the patients for providing information not available in the medical documents. We thank the medical staff for providing clinical experience.

Funding: This work was supported by the National Key Research and Development Program of China (2018YFA0108600); Natural Science Foundation of Beijing Municipality (7182134); CAMS Initiative for Innovative Medicine (2016-I2M-1-017) and Beijing Nova Program (Z181100006218003).

\section{Footnote}

Reporting Checklist: The authors have completed the STROBE reporting checklist. Available at http://dx.doi. org/10.21037/gs-21-49

Data Sharing Statement: Available at http://dx.doi. org/10.21037/.gs-21-47

Conflicts of Interest: All authors have completed the ICMJE uniform disclosure form (available at http://dx.doi. org/10.21037/gs-21-49). The author received fundings from the National Key Research and Development Program of China (2018YFA0108600), Natural Science Foundation of Beijing Municipality (7182134), CAMS Initiative for Innovative Medicine (2016-I2M-1-017), and Beijing Nova Program (Z181100006218003).

Ethical Statement: The authors are accountable for all aspects of the work in ensuring that questions related to the accuracy or integrity of any part of the work are appropriately investigated and resolved. The study was conducted in accordance with the Declaration of Helsinki (as revised in 2013). This study was approved by the Ethics Committee of PUMCH (JS-2288) and individual consent for this retrospective analysis was waived.

Open Access Statement: This is an Open Access article distributed in accordance with the Creative Commons Attribution-NonCommercial-NoDerivs 4.0 International License (CC BY-NC-ND 4.0), which permits the noncommercial replication and distribution of the article with the strict proviso that no changes or edits are made and the original work is properly cited (including links to both the formal publication through the relevant DOI and the license). See: https://creativecommons.org/licenses/by-nc-nd/4.0/.

\section{References}

1. Pivonello R, Isidori AM, De Martino MC, et al. Complications of Cushing's syndrome: state of the art. Lancet Diabetes Endocrinol 2016;4:611-29.

2. Hattori Y, Tahara S, Aso S, et al. Pituitary surgery's epidemiology using a national inpatient database in Japan. Acta Neurochir (Wien) 2020;162:1317-23.

3. Stroud A, Dhaliwal P, Alvarado R, et al. Outcomes of pituitary surgery for Cushing's disease: a systematic review and meta-analysis. Pituitary 2020;23:595-609.

4. Lonser RR, Nieman L, Oldfield EH. Cushing's disease: pathobiology, diagnosis, and management. J Neurosurg 2017;126:404-17.

5. Colao A, Pivonello R, Faggiano A, et al. Increased prevalence of thyroid autoimmunity in patients successfully treated for Cushing's disease. Clin Endocrinol (Oxf) 2000;53:13-9.

6. Onal ED, Sacikara M, Saglam F, et al. Primary thyroid disorders in patients with endogenous hypercortisolism: an observational study. Int J Endocrinol 2014;2014:732736.

7. Visser TJ, Lamberts SW. Regulation of TSH secretion and thyroid function in Cushing's disease. Acta Endocrinol (Copenh) 1981;96:480-3.

8. Benker G, Raida M, Olbricht T, et al. TSH secretion in Cushing's syndrome: relation to glucocorticoid excess, diabetes, goitre, and the 'sick euthyroid syndrome'. Clin Endocrinol (Oxf) 1990;33:777-86.

9. Brown DM, Lowman JT. Thyrotoxicosis occurring in two patients on prolonged high doses of steroids. N Engl J Med 1964;270:278-81.

10. Lamberg BA. Cushing's syndrome co-existing with hyperthyroidism. report of a case and some metabolic observations. Acta Med Scand 1964;175:Suppl 412:159+.

11. Kreines K, Esselborn VM. Simultaneous Graves' disease and Cushing's syndrome with unusually low levels of plasma cortisol. J Clin Endocrinol Metab 1968;28:789-94.

12. Kasperlik-Zatuska AA, Zgliczynski W. Increased prevalence of thyroid autoimmunity in patients successfully treated for Cushing's disease. Clin Endocrinol (Oxf) 2001;54:411; author reply 411.

13. Niepomniszcze H, Pitoia F, Katz SB, et al. Primary thyroid disorders in endogenous Cushing's syndrome. Eur J Endocrinol 2002;147:305-11.

14. Takasu N, Ohara N, Yamada T, et al. Development of autoimmune thyroid dysfunction after bilateral adrenalectomy in a patient with Carney's complex and after removal of ACTH-producing pituitary adenoma 
in a patient with Cushing's disease. J Endocrinol Invest 1993;16:697-702.

15. da Mota F, Murray C, Ezzat S. Overt immune dysfunction after Cushing's syndrome remission: a consecutive case series and review of the literature. J Clin Endocrinol Metab 2011;96:E1670-4.

16. Morita H, Isaji M, Mune T, et al. Transient Graves disease developing after surgery for Cushing disease. Am J Med Sci 2002;323:162-5.

17. Petramala L, Olmati F, Conforti MG, et al. Autoimmune Diseases in Patients with Cushing's Syndrome after Resolution of Hypercortisolism: Case Reports and Literature Review. Int J Endocrinol 2018;2018:1464967.

18. Tatsi C, Keil M, Lyssikatos C, et al. Incidence of Autoimmune and Related Disorders After Resolution of Endogenous Cushing Syndrome in Children. Horm Metab Res 2018;50:290-5.

19. Uthman I, Senécal JL. Onset of rheumatoid arthritis after surgical treatment of Cushing's disease. J Rheumatol 1995;22:1964-6.

20. Rehman HU, Walton C, Atkin S. Cushing's disease masking coincidental steroid-responsive diseases. Postgrad Med J 1999;75:43-5.

21. Noguchi Y, Tamai H, Fujisawa K, et al. Systemic lupus erythematosus after pituitary adenomectomy in a patient with Cushing's disease. Clin Endocrinol (Oxf) 1998;48:670-2.

22. Feng M, Liu ZY, Liu XH, et al. Diagnosis and Outcomes of 341 Patients with Cushing's Disease Following Transsphenoid Surgery: A Single-Center Experience. World Neurosurgery 2018;109:E75-E80.

23. Nieman LK, Biller BM, Findling JW, et al. Treatment of Cushing's Syndrome: An Endocrine Society

Cite this article as: Jiang S, Yang C, Feng M, Yao Y, Deng K, Xing B, Lu L, Zhu H, Wang R, Bao X. Management of thyrotoxicosis occurring after surgery for Cushing's disease: a case series. Gland Surg 2021;10(5):1627-1637. doi: 10.21037/gs21-49
Clinical Practice Guideline. J Clin Endocrinol Metab 2015;100:2807-31.

24. Effraimidis G, Strieder TG, Tijssen JG, et al. Natural history of the transition from euthyroidism to overt autoimmune hypo- or hyperthyroidism: a prospective study. Eur J Endocrinol 2011;164:107-13.

25. Bateman A, Singh A, Kral T, et al. The ImmuneHypothalamic-Pituitary-Adrenal Axis. Endocrine Reviews 1989;10:92-112.

26. Zilio M, Barbot M, Ceccato F, et al. Diagnosis and complications of Cushing's disease: gender-related differences. Clin Endocrinol (Oxf) 2014;80:403-10.

27. Effraimidis G, Wiersinga WM. Mechanisms in endocrinology: autoimmune thyroid disease: old and new players. Eur J Endocrinol 2014;170:R241-52.

28. Efstathiadou ZA, Sykja A, Anagnostis P, et al. Occurrence of De Quervain's Thyroiditis after Resolution of Hypercortisolism following Pasireotide Treatment for Cushing's Disease and Surgery for an Adrenocortical Adenoma: Report of Two Cases. Eur Thyroid J 2014;3:69-72.

29. Snyder NJ, Green DE, Solomon DH. GlucocorticoidInduced Disappearance of Long-Acting Thyroid Stimulator in the Ophthalmopathy of Graves' Disease. J Clin Endocrinol Metab 1964;24:1129-35.

30. Pearce EN, Farwell AP, Braverman LE. Thyroiditis. N Engl J Med 2003;348:2646-55.

31. Ross DS. Exogenous hyperthyroidism. In: Mulder JE, editor. UpToDate. UpToDate, Waltham, MA. 2019.

32. Yamakita N, Sakata S, Hayashi H, et al. Case report: silent thyroiditis after adrenalectomy in a patient with Cushing's syndrome. Am J Med Sci 1993;305:304-6. 
Supplementary

Table S1 Characteristics of 14 patients before surgery for Cushing's disease (CD)

\begin{tabular}{|c|c|c|c|c|c|c|c|c|c|c|}
\hline $\mathrm{Pt}$ & $\begin{array}{l}\text { Age at CD } \\
\text { diagnosis }\end{array}$ & Sex & $\begin{array}{c}\text { Disease duration } \\
\text { (years) }\end{array}$ & $\begin{array}{l}\text { Tumor size } \\
\qquad\left(\mathrm{mm}^{3}\right)\end{array}$ & Thyroid USG & $\begin{array}{c}\text { TSH } \\
(\mu \mathrm{lU} / \mathrm{mL})\end{array}$ & $\begin{array}{c}\text { FT3 } \\
\text { (pg/mL) }\end{array}$ & $\begin{array}{c}\text { FT4 } \\
\text { (ng/dL) }\end{array}$ & TPOAb & $\operatorname{TgAb}$ \\
\hline 1 & 33 & $\mathrm{~F}$ & 3 & $8^{*} 8^{*} 6$ & Normal & 2.237 & 2.83 & 1.047 & neg & neg \\
\hline 3 & 28 & $\mathrm{~F}$ & 3 & $10^{\star} 8 * 8$ & & 1.335 & 2.00 & 1.233 & & \\
\hline 4 & 80 & $\mathrm{~F}$ & 5 & $20 * 32 * 18$ & & 1.183 & 1.37 & 0.691 & & \\
\hline 6 & 45 & $\mathrm{~F}$ & 8 & $5^{*} 3^{*} 3$ & $\mathrm{D}$ & 1.379 & 2.76 & 1.126 & neg & neg \\
\hline 7 & 23 & $\mathrm{~F}$ & 1.6 & $6^{*} 6^{*} 6$ & $\mathrm{D}$ & 1.935 & 2.93 & 1.230 & neg & neg \\
\hline 8 & 32 & $\mathrm{~F}$ & 4 & $5 * 5 * 5$ & & 0.798 & 2.56 & 0.983 & & \\
\hline 9 & 16 & $\mathrm{~F}$ & 1.5 & $4^{\star} 5^{\star} 6$ & D & 1.390 & 2.96 & 1.128 & & \\
\hline 13 & 29 & $\mathrm{~F}$ & 3 & $5^{\star} 5^{\star} 4$ & & 0.746 & 2.57 & 1.223 & & \\
\hline 14 & 35 & $\mathrm{~F}$ & 8 & $8^{*} 7^{*} 6$ & & 1.099 & 2.82 & 1.180 & neg & neg \\
\hline
\end{tabular}

D thyroid with normal size but with nodules. neg, negative; USG, ultrasonography; TPOAb, anti-thyroid peroxidase antibody; TgAb, antithyroglobulin antibody; FT4, free thyroxine; FT3, free triiodothyronine; TSH, thyroid stimulating hormone; Hormonal normal values, FT4: $0.81-1.89 \mathrm{ng} / \mathrm{dL}$; FT3: $1.8-4.1 \mathrm{pg} / \mathrm{mL}$; TSH: $0.38-4.34 \mu \mathrm{lU} / \mathrm{mL}$. The patients are numbered according to the time when their surgeries were performed.

Table S2 Clinical profiles of 14 patients with postoperative thyrotoxicosis after surgery for Cushing's disease

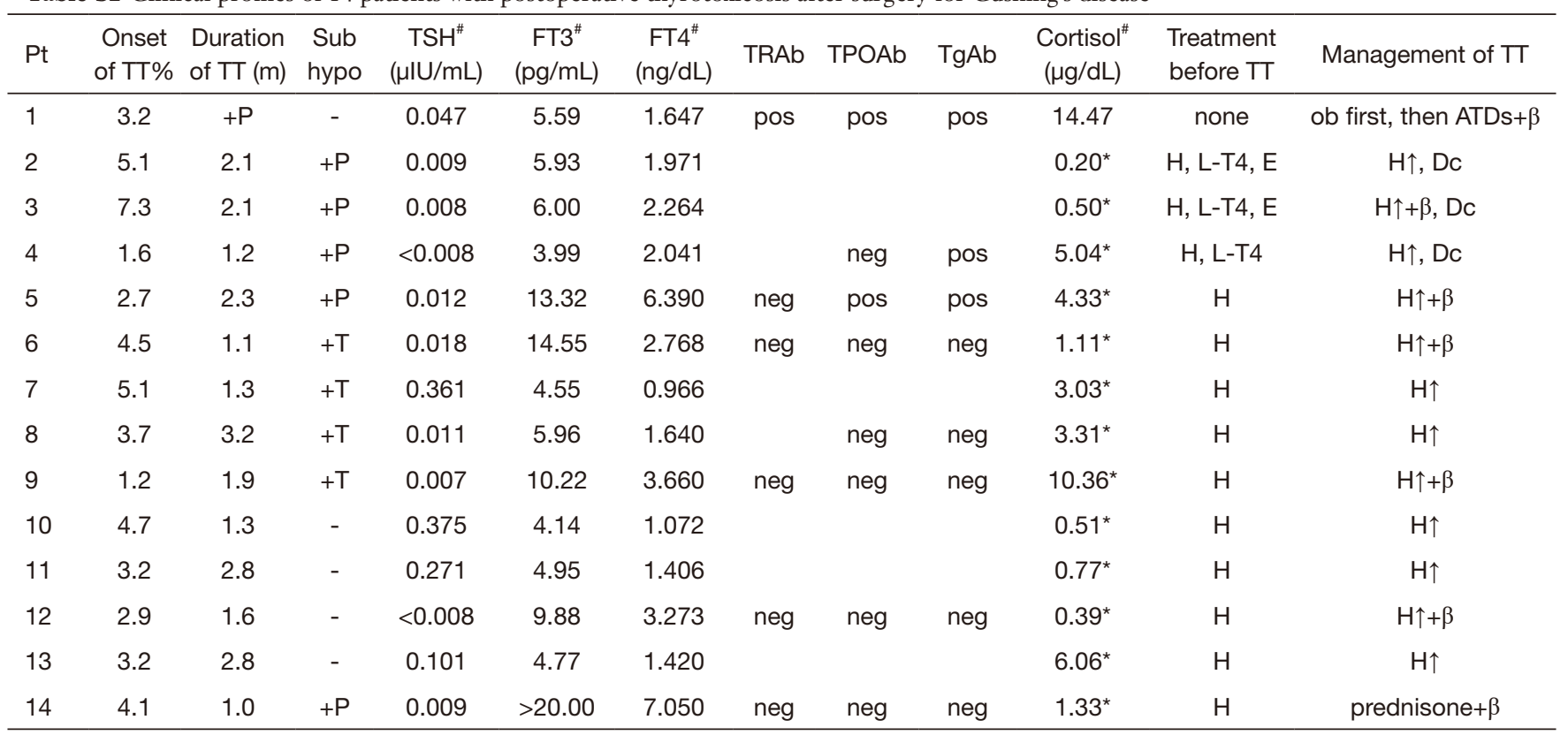

$\%$ months after surgery; ${ }^{\text {" }}$ tested at the time of diagnosis of thyrotoxicosis; ${ }^{*}$ tested before the morning hydrocortisone administration; $\Pi$, thyrotoxicosis; Sub hypo, subsequent hypothyroidism; pos, positive; neg, negative; $+\mathrm{P}$, permanent; $+\mathrm{T}$, transient; $\mathrm{H}$, hydrocortisone; $\mathrm{H} \uparrow$, higher dose of hydrocortisone; TRAb, anti-thyrotropin receptor antibody; - no subsequent hypothyroidism; L-T4, levothyroxine; Dc, discontinue levothyroxine; $\beta$, $\beta$-blockers; ATDs, anti-thyroid drugs; E, estrogens. Hormonal normal values, FT4: 0.81-1.89 ng/dL; FT3: 1.8-4.1 pg/mL; TSH: 0.38-4.34 $\mu \mathrm{lU} / \mathrm{mL}$, cortisol: 4.0-22.3 $\mu \mathrm{g} / \mathrm{dL}$. 